\title{
DESEMPENHO EM TESTE CARDIOPULMONAR DE ADOLESCENTES: PESO NORMAL E EXCESSO DE PESO
}

\author{
PERFORMANCE ON CARDIOPULMONARY TESTING IN ADOLESCENTS: NORMAL \\ WEIGHT AND OVERWEIGHT \\ DESEMPEÑO EN PRUEBA CARDIOPULMONAR DE ADOLESCENTES CON PESO \\ NORMAL Y SOBREPESO
}

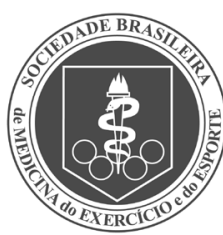

Artigo Original

\begin{abstract}
Kamilla Bolonha Gomes' (Professora de Educação Física) Luciana Carletti

(Professora de Educação Física)

Anselmo José Perez ${ }^{2}$

(Professor de Educação Física)

1. Programa de Orientação ao Exercício Físico, Prefeitura Municipal de Serra, Serra, ES, Brasil. 2. Universidade Federal do Espírito Santo (UFES), Vitória, ES, Brasil.
\end{abstract}

\section{Correspondência:}

Rua Antiocho Carneiro de Mendonça, 170, ap. 807, Jardim Camburi, 29092-130. Vitória, ES, Brasil. kamillabolonha@yahoo.com.br

\section{RESUMO}

Introdução: Sabe-se que o excesso de peso interfere no desempenho físico do cotidiano do adolescente e, consequentemente, na aptidão física e na capacidade cardiorrespiratória. Objetivo: O estudo objetivou identificar e comparar os valores das variáveis cardiorrespiratórias no limiar anaeróbico ventilatório (LAV) de adolescentes com peso normal e excesso de peso. Métodos: A amostra foi composta por 140 adolescentes de ambos os sexos entre 10 e 14 anos, escolares do município de Vitória, ES, Brasil. Foram mensuradas massa corporal e estatura para cálculo do índice de massa corporal (IMC). Os adolescentes foram divididos em grupo peso normal (GPN) e grupo excesso de peso (GEP), realizaram um eletrocardiograma de repouso e um teste cardiopulmonar de exercício em esteira ergométrica (Inbrasport Super ATL) utilizando o ergoespirômetro MedGraphics Corporation e o protocolo de rampa. O LAV foi identificado pelo método V-slope e/ou equivalente ventilatório de oxigênio $\left(\mathrm{VEN} \mathrm{NO}_{2}\right)$. Foram analisadas as seguintes variáveis no LAV: consumo de oxigênio $\left(\mathrm{VO}_{2 L \mathrm{AV}} \mathrm{ml}^{\mathrm{kgg}} \mathrm{kg}^{-1} \cdot \mathrm{min}^{-1}\right)^{-1}$, frequência cardíaca ( $\left.\mathrm{FC}_{\text {LAV }} \mathrm{bpm}\right)$, percentuais do consumo máximo de oxigênio $\left(\% \mathrm{VO}_{2 \text { máxLAVV }}\right)$ e da $\mathrm{FC}$ máxima $\left(\% F C_{\text {máxLAVV }}\right)$, e velocidade $\left(V e l_{L A V} \mathrm{~km} / \mathrm{h}\right)$, além do consumo máximo de oxigênio $\left(V_{\mathrm{O}_{\text {máx }}} \mathrm{ml} \cdot \mathrm{kg}^{-1} \cdot \mathrm{min}^{-1}\right)$ e $F C$ máxima $\left(F C_{\text {máx }}\right)$. Foi realizado teste $t$ de Student nas comparações entre os grupos, considerando $p<0,05$ para significância estatística. Resultados: O GPN apresentou valores significativamente maiores no $\mathrm{LAV}_{\text {para }} \mathrm{VO}_{2} \mathrm{ml}_{\mathrm{kg}} \mathrm{kg}^{-1} \mathrm{~min}^{-1}(20,0 \pm 6,4$ vs $15,8 \pm 5,7)$ e velocidade $(7,1 \pm 0,9 \mathrm{~km} / \mathrm{h}$ vs $6,2 \pm 1,1 \mathrm{~km} / \mathrm{h})$, e para o $\mathrm{VO}_{2 \text { máx }}(44,6 \pm 7,6 \mathrm{vs} 36,0 \pm 6,4)$ quando comparado ao GEP. Conclusão: Os dados sugerem que adolescentes com excesso de peso apresentam prejuízo na aptidão cardiorrespiratória não somente ao nível máximo, mas também ao nível submáximo (LAV) quando comparados aos adolescentes com peso normal.

Palavras-chave: consumo de oxigênio, adolescente, aptidão física, limiar anaeróbio.

\section{ABSTRACT}

Introduction: It is known that excess weight interferes with the daily physical performance of adolescents and consequently, in physical adaptation and cardiorespiratory capacity. Objective: The study aimed to identify and compare the cardiorespiratory variables at the ventilatory anaerobic threshold (VAT) of normal weight and overweight adolescents. Methods: The sample consisted of 140 adolescent students of both sexes, aged between 10 and 14 years, in the city of Vitória, ES. Body weight and height were measured, to calculate the body mass index (BMI). The adolescents were divided into normal weight group (NWG) and overweight group (OWG). A resting electrocardiogram was performed, and a cardiopulmonary exercise test on a treadmill (Inbrasport Super ATL) using a MedGraphics Corporation ergospirometer and the ramp protocol. The VAT was identified by the V-slope method and/or ventilatory equivalent of oxygen $\left(V_{E} \mathrm{NO}_{2}\right)$. The following variables were analyzed in the VAT: oxygen consumption $\left(\mathrm{VO}_{2 V A T} \mathrm{ml} . \mathrm{kg}^{-1} \cdot \mathrm{min}^{-1}\right)$, heart rate (bpm HR $\left.R_{V A T}\right)$, percentages of maximum oxygen uptake $\left(\% \mathrm{VO}_{2 \text { maxVAT }}\right)$ and maximum $H R\left(\% H R_{\text {maxVAT }}\right)$ and speed (Vel $\left.V_{V A T} \mathrm{~km} / \mathrm{h}\right)$, as well as maximum oxygen uptake $\left(\mathrm{VO}_{2 \max } \mathrm{ml}^{\mathrm{kg}} \mathrm{kg}^{-1} \cdot \mathrm{min}^{-1}\right)$ and maximum heart rate $\left(H R_{\max }\right)$. The Student's T-test was performed for comparisons between groups, considering $p<0.05$ for statistical significance. Results: The NWG was significantly higher in VAT for VO $\mathrm{ml}^{\mathrm{kg}} \mathrm{kg}^{-1} \mathrm{~min}^{-1}(20.0 \pm 6.4 \mathrm{vs} 15.8 \pm 5.7)$ and speed $\left(7.1 \pm 0.9 \mathrm{~km} / \mathrm{h}\right.$ vs $6.2 \pm 1.1 \mathrm{~km} / \mathrm{h}$ ), and $\mathrm{VO}_{2 \max }(44.6 \pm 7.6 \mathrm{vs} .36 .0 \pm 6.4$ ) compared to the OWG. Conclusion: The data suggest that overweight adolescents have impaired cardiorespiratory fitness not only at the maximum level, but also at submaximal level (VAT), compared with normal weight adolescents.

Keywords: oxygen consumption, adolescent, physical fitness, anaerobic threshold.

\section{RESUMEN}

Introducción: Se sabe que el exceso de peso interfiere con el desempeño físico cotidiano del adolescente y por lo tanto en la aptitud física y la capacidad cardiorrespiratoria. Objetivo: El objetivo del estudio fue identificar y comparar los valores de las variables cardiorrespiratorias en el umbral anaerobio ventilatorio (UAV) de los adolescentes con peso normal y sobrepeso. Métodos: La muestra estuvo conformada por 140 adolescentes de ambos sexos entre 10 y 14 años, estudiantes de la ciudad de Vitória/ES. Se midieron el peso corporal y la altura para calcular el índice de masa corporal (IMC). Los adolescentes fueron divididos en grupo de peso normal (GPN) y grupo de exceso de peso (GEP); 
se realizó un electrocardiograma en reposo y prueba de esfuerzo cardiopulmonar en una caminadora (Inbrasport Super ATL), utilizándose ergoespirómetro MedGraphics Corporation y el protocolo de rampa. EI UAV fue identificado por el método de la V-slope y/o equivalente ventilatorio de oxígeno $\left(V E N \mathrm{~N}_{2}\right)$. Las siguientes variables fueron analizadas en el UAV: consumo de oxígeno $\left(V_{2} \mathrm{ZuV}_{\mathrm{V}} \mathrm{ml} \cdot \mathrm{kg}^{-1} . \mathrm{min}^{-1}\right)$, la frecuencia cardiaca $\left(F C_{\text {Uav }} / \mathrm{pm}\right)$, el porcentaje de

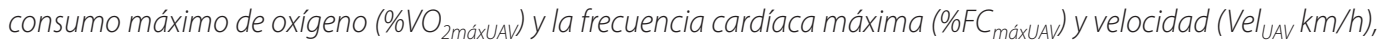
y el consumo máximo de oxígeno $\left(\mathrm{VO}_{2 \text { máx }} \mathrm{ml} \cdot \mathrm{kg}^{-1} \cdot \mathrm{min}^{-1}\right)$ y la frecuencia cardíaca máxima $\left(F C_{\text {máx }}\right)$. La prueba $t$ de Student se realizó para las comparaciones entre grupos, considerándose $p<0,05$ para la significación estadística. Resultados: El GPN tuvo UAV significativamente mayor para VO $\mathrm{ml}^{\mathrm{kg}}$. $\mathrm{min}^{-1}(20,0 \pm 6,4 \mathrm{vs} .15,8 \pm 5,7)$ y velocidad $(7,1 \pm 0,9 \mathrm{~km} / \mathrm{h}$ vs. 6,2 $\pm 1,1 \mathrm{~km} / \mathrm{h})$ y el VO $\mathrm{V}_{2 \text { máx }}(44,6 \pm 7,6 \mathrm{vs}$. 36,0 $\pm 6,4)$ en comparación con el GEP. Conclusión: Los datos sugieren que los adolescentes con sobrepeso tienen problemas de capacidad cardiorrespiratoria no sólo en el nivel máximo, sino también a nivel submáximo (UAV) en comparación con los adolescentes de peso normal.

Palabras clave: consumo de oxígeno, adolescente, aptitud física, umbral anaerobio.

\section{INTRODUÇÃO}

Considerado um problema de saúde pública, o excesso de peso tem caráter epidemiológico global em todas as faixas etárias, segundo os dados da Organização Mundial da Saúde1 (OMS). No Brasil sua frequência triplicou a partir do final da década de 1980, diagnosticado em cerca de um quinto dos adolescentes (entre 10 e 19 anos), sendo a obesidade correspondente a um quarto do total de casos de excesso de peso ${ }^{2}$.

Sabe-se que o excesso de peso interfere no desempenho físico do cotidiano do adolescente e, consequentemente, na aptidão física e na capacidade cardiorrespiratória ${ }^{3-7}$. Sendo assim, alguns autores ${ }^{6-9}$ apontam que o aumento da massa de gordura corporal reduz o consumo máximo de oxigênio de adolescentes obesos, com valores de aptidão cardiorrespiratória mais baixos quando comparados aos indivíduos com peso normal.

Para analisar o desempenho na intensidade submáxima do esforço, que é um indicativo da aptidão cardiorrespiratória, o limiar anaeróbico ventilatório (LAV) tem sido muito utilizado por se tratar de um parâmetro da resposta metabólica relacionada ao aumento progressivo da carga de trabalho, observada à partir da análise da cinética das trocas gasosas, utilizando o teste cardiopulmonar de exercício com carga progressiva. Por ser o LAV um parâmetro não invasivo, este permite o maior desenvolvimento de pesquisas relacionadas com a aptidão cardiorrespiratória, principalmente em crianças e adolescentes. Porém, observa-se que são escassos os trabalhos que estudam adolescentes (10 - 14 anos) com excesso de peso ao nível do desempenho submáximo nesse tipo de teste utilizando o LAV como referência.

$\mathrm{Na}$ literatura alguns estudos mostram que o $\mathrm{VO}_{2}$ no LAV se correlaciona bem com o $\mathrm{VO}_{2 \text { máx }}$ em crianças e adolescentes, e que tem sido utilizado como um parâmetro de avaliação da aptidão cardiorrespiratória do exercício submáximo nessa população 3,4,10,11. O LAV é também sugerido para utilização em populações com excesso de peso, pois além de ser um método não invasivo das alterações acido-básico mensuradas pelas trocas gasosas durante o exercício, não requer um teste máximo, sendo importante para indivíduos com alguma intolerância ao exercício ${ }^{7,11}$.

A partir do exposto, a identificação das variáveis cardiorrespiratórias ao nível do LAV se torna importante para o entendimento da resposta ventilatória e análise da aptidão cardiorrespiratória tanto relacionada à saúde, uma vez que uma maior aptidão pode estar correlacionada a um menor perfil de risco cardiovascular ${ }^{12,13}$, como à prescrição de exercícios e ao efeito do treinamento ${ }^{6,14}$ nesta população, com ou sem excesso de peso de ambos os gêneros, o qual poderá auxiliar na prescrição de exercícios físicos em intensidades mais apropriadas para essa população.
Neste sentido, o estudo objetivou identificar e comparar os valores das variáveis cardiorrespiratórias no limiar anaeróbico ventilatório (LAV) de adolescentes com peso normal e excesso de peso.

\section{MATERIAL E MÉTODOS}

Neste estudo estão reunidos dados que foram coletados entre 2003 e 2005, por meio de convênio científico entre a Faculdade Salesiana de Vitória e a Universidade Federal do Espírito Santo (UFES). A amostra foi composta por 140 adolescentes escolares, de ambos os sexos, entre 10 e 14 anos, que foi obtida por um processo de seleção aleatória, considerando a proporcionalidade entre a população dessa faixa etária nas sete regiões administrativas do município de Vitória, ES, Brasil.

As escolas de cada uma das sete regiões administrativas foram sorteadas e convidadas a participar do estudo, por meio de seus diretores, que forneceram uma listagem de seus alunos. A partir destas listas os escolares foram sorteados de maneira aleatória, onde apenas dois deles desistiram de participar, os quais foram substituídos através de novo sorteio. Os adolescentes sorteados foram convidados a participar do estudo por meio de seus pais ou responsáveis, que assinaram o termo de consentimento livre e esclarecido sobre os benefícios, riscos e procedimentos a serem realizados.

Foram considerados como critérios de exclusão: ser tabagista ou portador de alguma doença metabólica previamente conhecida.

A idade cronológica dos adolescentes foi determinada em forma centesimal, tendo como referência a data da coleta de dados e a data do nascimento.

O protocolo de estudo foi aprovado pelo Comitê de Ética em Pesquisa da Faculdade Salesiana de Vitória, por meio do Ofício 069/2007 - CEP/FSV.

Medidas antropométricas e divisão dos grupos: A massa corporal foi determinada utilizando uma balança antropométrica (Welmy, modelo RW200; São Paulo, BR) com precisão de 100 gramas (g) e capacidade máxima para 150 quilos (kg), e a estatura foi aferida utilizando um estadiômetro de madeira (Seca, modelo 206) com escala de 0,1 centímetros (cm). A partir dessas duas variáveis, calculou-se o Índice de Massa Corporal (IMC, kg/m²).

Os adolescentes foram divididos em grupos de acordo com o índice de massa corporal (IMC), a partir dos valores de referência propostos pela Organização Mundial da Saúde ${ }^{15}$, que utiliza os valores médios de IMC para idade e sexo, estabelecendo como ponto de corte o percentil $>85$ e $\leq 97$ para sobrepeso e > 97 para obesidade.

Sendo assim, os adolescentes foram divididos em dois grupos: peso normal (GPN; percentil < 85) e excesso de peso (GEP, composto pela classificação sobrepeso e obesidade). 


\section{Protocolo do teste de esforço cardiopulmonar}

Inicialmente, os adolescentes realizaram um eletrocardiograma (ECG) de repouso utilizando as 12 derivações convencionais. Eles permaneciam em decúbito dorsal por cerca de cinco minutos, em um ambiente tranquilo, com temperatura mantida em torno de $22^{\circ} C_{\text {; a }}$ seguir, era feito o registro da atividade elétrica do coração. Em seguida, eram encaminhados para a esteira ergométrica e orientados a respeito do desenvolvimento do teste cardiopulmonar de exercício e os critérios para a interrupção do mesmo.

Foi utilizada uma esteira ergométrica Inbrasport Super ATL (RS, Brasil) e um ergoespirômetro $\mathrm{CardiO}_{2}$ MedGraphics Corporation (modelo (PX/D; Minnesota, EUA), o qual fornecia informações sobre o consumo de oxigênio $\left(\mathrm{VO}_{2}\right)$, produção de dióxido de carbono $\left(\mathrm{VCO}_{2}\right)$, ventilação pulmonar (VE), equivalente ventilatório de oxigênio $\left(\mathrm{VE} / \mathrm{VO}_{2}\right)$ e equivalente ventilatório de dióxido de carbono $\left(\mathrm{VE} / \mathrm{NCO}_{2}\right)$ e razão de troca respiratória $\left(\mathrm{RTR}=\mathrm{VCO}_{2} \mathrm{NO}_{2}\right)$.

Uma máscara de neoprene conectada ao pneumotacômetro para medida do fluxo de ar e análise dos gases expirados foi utilizada de acordo com o tamanho do rosto de cada adolescente avaliado, permitindo a respiração pela boca e pelo nariz.

O teste era iniciado após dois minutos de repouso em pé realizando os registros eletrocardiográficos e ventilatórios. Durante a execução, os indivíduos eram monitorados por meio do ECG de 12 derivações (Elite/Micromed, Brasília, DF, Brasil), para acompanhar a resposta cardíaca e a frequência cardíaca (FC) do esforço. Foi aplicado o protocolo de rampa, que consiste no aumento progressivo da carga de trabalho (velocidade, em $\mathrm{km} / \mathrm{h}$ e inclinação da esteira, em percentagem) de acordo com o $\mathrm{VO}_{2}$ predito em equivalentes metabólicos (MET), comparado com o $\mathrm{VO}_{2}$ medido.

Quando o adolescente atingia a velocidade máxima predita sem que estivesse em fadiga, a inclinação era aumentada com incremento gradual de 0,5\% a cada 45 segundos. O teste era interrompido se o avaliado sinalizasse (através de gestos pré-combinados) para a exaustão, ou algum desconforto que o impedisse na continuidade do mesmo.

Os testes foram considerados máximos quando ${ }^{16}:$ a) $\mathrm{FC}_{\text {máx }}$ atingida > 190 bpm; e b) RTR > 1,0.

O ergoespirômetro consiste em um sistema de calorimetria de circuito aberto, ou seja, o gás de referência para calibração era ajustado por uma mistura de gases com concentração constante de $\mathrm{CO}_{2}$ e $\mathrm{O}_{2}$. Um software "breeze suíte" foi utilizado para determinação da concentração do $\mathrm{VO}_{2}$ e VCO $\mathrm{na} V \mathrm{~V}$, pela medida da diferença entre as pressões gasosas no ar inspirado e no ar expirado, aferidos a cada respiração. Um transdutor fazia quantificação da concentração do $\mathrm{CO}_{2}$ através de análise infravermelha não dispersiva (NDIR), e outro fazia a quantificação do $\mathrm{O}_{2}$ por meio de célula de zircônio.

O equipamento era calibrado antes do teste: aguardava-se $30 \mathrm{mi}-$ nutos para o aquecimento do circuito; em seguida, ajustava-se o fluxo de ar através do uso de uma seringa de calibração com capacidade de gerar um volume de três litros $(L)$; a seringa era manipulada manualmente para produzir um fluxo de 0,4 a $12 \mathrm{~L} / \mathrm{s}$ para verificar a calibração do sistema para fluxo baixo e alto; o sensor de $\mathrm{O}_{2}$ e $\mathrm{CO}_{2}$ era calibrado usando como referência um gás composto de $22 \%$ de $\mathrm{O}_{2}$ e $0 \%$ de $\mathrm{CO}_{2}$; finalmente, o sensor era calibrado com o uso de um gás composto de $12 \%$ de $\mathrm{O}_{2}$ e $5 \%$ de $\mathrm{CO}_{2}$.

Identificação do Limiar Anaeróbico Ventilatório (LAV): O LAV foi identificado visualmente por dois avaliadores, utilizando o método V-slope (relação $\mathrm{VCO}_{2}$ vs $\mathrm{VO}_{2}$ ) e/ou equivalente ventilatório de oxigênio (VE/ $\mathrm{VO}_{2}$ ), de acordo com os seguintes critérios ${ }^{17}$ : ponto de quebra da linearidade da inclinação de $\mathrm{VCO}_{2} \mathrm{VSVO}_{2}$ (método $\mathrm{V}$-slope), e/ou o aumento no VENO $\mathrm{N}_{2}$ sem aumento concomitante no VEN $\mathrm{NCO}_{2}$.

\section{Análise estatística}

Para as comparações entre os grupos das variáveis $V_{2} \mathrm{Z}_{2 \perp A V}, V_{2 \text { máx }}$

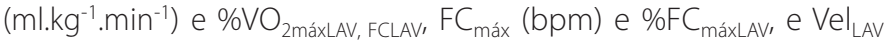
$(\mathrm{km} / \mathrm{h})$ foram utilizados a estatística descritiva (média, desvio padrão) e o teste $t$ de Student para amostras independentes, considerando $p<$ 0,05 para significância estatística. Os cálculos foram realizados utilizando o software SigmaStat, versão 3.5 .

\section{RESULTADOS}

Foram analisados os dados de 140 adolescentes, os quais atenderam aos critérios para o máximo do teste cardiopulmonar de exercício e para a identificação do LAV. Os participantes tinham idades entre 10 e 14 anos, sendo 42 meninas e 65 meninos compondo o grupo de peso normal (GPN); 13 meninas e 20 meninos compondo o grupo de excesso de peso (GEP).

As características antropométricas e médias de idade dos grupos são apresentadas na tabela 1. Como esperado, houve diferença estatística nos valores de massa corporal, estatura e IMC, sendo GEP > GPN.

Na tabela 2, estão os valores da aptidão cardiorrespiratória e da velocidade que foram comparados entre adolescentes com peso normal e excesso de peso. As variáveis analisadas e comparadas no LAV foram: $V O_{2 \perp A V}\left(\mathrm{ml}^{\prime} \mathrm{kg}^{-1} \cdot \mathrm{min}^{-1}\right), \% \mathrm{VO}_{2 \text { máx LAV }}, \mathrm{FC}_{\text {LAV }}(\mathrm{bpm}), \% \mathrm{FC}_{\text {máxLAV }}$ e velocidade $\left(V_{\text {el }} \mathrm{LAV}_{\mathrm{k}} \mathrm{km} / \mathrm{h}\right)$. Entre as variáveis no esforço máximo do teste estão: $\mathrm{VO}_{2 \text { máx }}\left(\mathrm{ml} . \mathrm{kg}^{-1} \cdot \mathrm{min}^{-1}\right)$ e $\mathrm{FC}_{\text {máx }}(\mathrm{bpm})$.

É possível observar que tanto o $\mathrm{VO}_{2 L \mathrm{AV}}\left(\mathrm{ml} \mathrm{kg}^{-1} \cdot \mathrm{min}^{-1}\right)$ quanto o $\mathrm{VO}_{2 \text { máx }}$ $\left(\mathrm{ml} . \mathrm{kg}^{-1} \cdot \mathrm{min}^{-1}\right)$ foram significativamente maiores para GPN quando comparados com GEP (figura 1). Porém, mesmo encontrando diferenças entre os grupos nas variáveis referentes ao consumo de $\mathrm{O}_{2}$ (máximo e submáximo), onde são vistos os menores valores no GEP, foi observada uma semelhança estatística do \% $\mathrm{VO}_{2 \text { máx LAv }}$ entre os eles $(p=0,562)$.

Quanto às variáveis relacionadas à frequência cardíaca $\left(\mathrm{FC}_{\mathrm{LAV}}, \mathrm{FC}_{\text {máx }}\right.$ e \% F( $\left.C_{\text {máxLLAv }}\right)$ não foram observadas diferenças estatísticas significantes entre os grupos ( $p=0,799 ; p=0,121 ; p=0,237$, respectivamente).

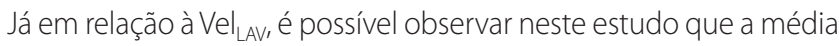
do GPN é significativamente maior do que a média do GEP, sendo de $7,1 \mathrm{~km} / \mathrm{h}$ e $6,2 \mathrm{~km} / \mathrm{h}$, respectivamente.

Tabela 1. Características antropométricas dos grupos estudados.

\begin{tabular}{|c|c|c|c|c|}
\hline & \multicolumn{2}{|c|}{ GPN } & \multicolumn{2}{|c|}{ GEP } \\
\hline & \multicolumn{2}{|c|}{$(n=107)$} & \multicolumn{2}{|c|}{$(n=33)$} \\
\hline Idade (anos) & 12,1 & $\pm 1,2$ & 11,6 & $\pm 0,9$ \\
\hline Massa corporal (kg) & 35,7 & $\pm 6,8$ & 54,6 & $\pm 11,2^{*}$ \\
\hline Estatura (m) & 1,46 & $\pm 0,08$ & 1,51 & $\pm 0,06^{*}$ \\
\hline IMC $\left(\mathrm{kg} / \mathrm{m}^{2}\right)$ & 16,6 & $\pm 1,8$ & 23,8 & $\pm 3,3^{*}$ \\
\hline
\end{tabular}
Student: ${ }^{*} p<0,05$

Tabela 2. Comparação da aptidão cardiorrespiratória e velocidade dos grupos estudados.

\begin{tabular}{|c|c|c|}
\hline & GPN & GEP \\
\hline $\mathrm{VO}_{2 \text { máx }}\left(\mathrm{ml} \mathrm{kg}^{-1} \cdot \mathrm{min}^{-1}\right)$ & $44,6 \pm 7,6^{*}$ & $36,0 \pm 6,4$ \\
\hline $\mathrm{VO}_{2\llcorner\mathrm{AV}}\left(\mathrm{ml} \cdot \mathrm{kg}^{-1} \cdot \mathrm{min}^{-1}\right)$ & $20,0 \pm 6,4^{*}$ & $15,8 \pm 5,7$ \\
\hline$\% \mathrm{VO}_{2 \text { máxLAV }}$ & $45,3 \pm 13,6$ & $45,3 \pm 13,6$ \\
\hline$F C_{\text {máx }}(\mathrm{bpm})$ & $198 \pm 7$ & $201 \pm 9$ \\
\hline $\mathrm{FC}_{\text {LAV }}(\mathrm{bpm})$ & $139 \pm 20$ & $138 \pm 17$ \\
\hline$\% \mathrm{FC}_{\text {máxlaV }}$ & $70,1 \pm 10$ & $69,0 \pm 8$ \\
\hline $\operatorname{Vel}_{\text {LAV }}(\mathrm{km} / \mathrm{h})$ & $7,1 \pm 0,9^{*}$ & $6,2 \pm 1,1$ \\
\hline
\end{tabular}




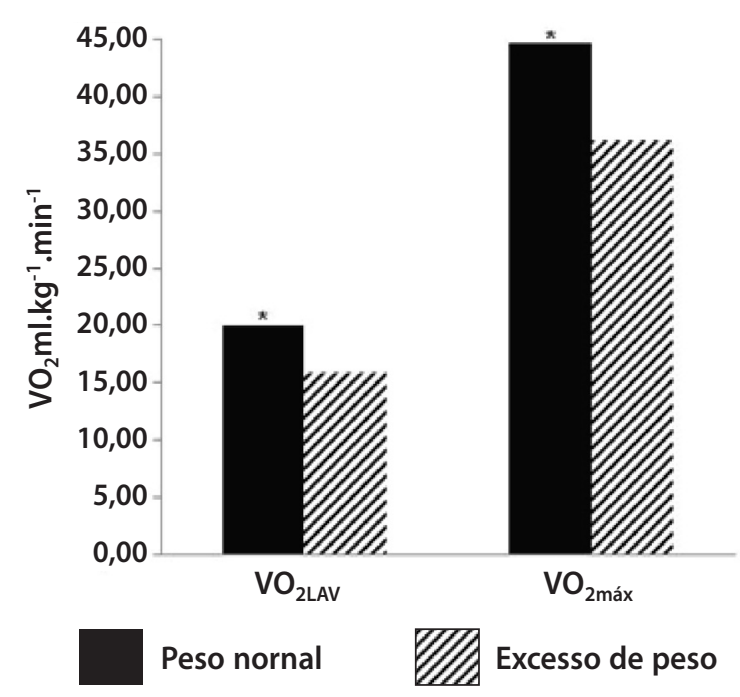

Teste $t$ de Student: * $p<0,05 ;$ GPN: peso normal; GEP: excesso de peso.

Figura 1. Valores médios do consumo de oxigênio no LAV $\left(V_{2} O_{A V}\right)$ e máximo $\left(V_{2 m a ́ x}\right)$ do teste cardiopulmonar de exercício.

\section{DISCUSSÃO}

A proposta deste estudo foi identificar e comparar os valores das variáveis cardiorrespiratórias no LAV entre os grupos com peso normal e com excesso de peso, em adolescentes de ambos os sexos. Assim, de uma maneira geral, os adolescentes com excesso de peso apresentaram uma aptidão cardiorrespiratória prejudicada, em nível submáximo de desempenho no teste cardiopulmonar de exercício, quando comparados aos adolescentes com peso normal.

Neste estudo, optamos por denominar apenas dois grupos (peso normal e excesso de peso) tomando por base os resultados de um estudo realizado por Gomes et al.18. Na dissertação citada foram analisados e comparados três grupos (peso normal, sobrepeso e obesidade) cujos resultados do grupo com sobrepeso foram semelhantes ao grupo com obesidade para as variáveis cardiorrespiratórias no LAV, concluindo-se que o início da presença do excesso de peso no adolescente já poderia trazer prejuízos para o seu desempenho. Sendo assim, foi possível agregar os adolescentes dos grupos sobrepeso e obesidade e chamá-los de excesso de peso.

Ao analisar as respostas do $\mathrm{VO}_{2\llcorner A V} \in \mathrm{VO}_{2 \text { máx }}$ ambos relativos a massa corporal, os adolescentes com excesso de peso apresentaram valores menores quando comparados ao GPN (GEP: VO ${ }_{2 \text { LAV }} 15,8 \pm 5,7$ e VO 2máx $36,0 \pm 6,4 ; G P N: V_{2 L A V} 20,0 \pm 6,4$ e VO$\left._{2 \text { máx }} 44,6 \pm 7,6\right)$, o que pode ser explicado, em parte, pela maior massa corporal que os adolescentes com excesso de peso apresentam, ou pela baixa aptidão cardiorrespiratória

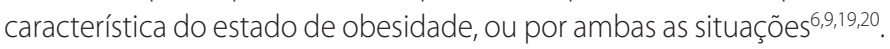
Além disso, Bar-Or ${ }^{21}$ relata que crianças com obesidade apresentam desempenho deficiente na atividade física quando comparadas às crianças magras, e uma razão para isso é o alto custo metabólico do exercício, onde os obesos requerem maior captação de $\mathrm{O}_{2}$ para executar determinada tarefa.

Embora não tenham sido encontradas diferenças entre os grupos

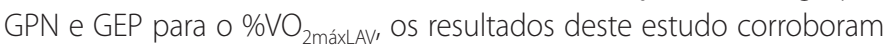
com a literatura ${ }^{7}$, onde os autores mostraram que o $\mathrm{VO}_{2 \text { máx }}\left(\mathrm{ml}^{\mathrm{kg}} \mathrm{kg}^{-1} \cdot \mathrm{min}^{-1}\right)$ e o VO ${ }_{2 L A V}\left(\mathrm{mllkg}^{-1} \cdot \mathrm{min}^{-1}\right)$ foram significativamente menores nos obesos do que naqueles com peso normal, mas não havendo diferenças significativas entre os grupos para valores absolutos do $\mathrm{VO}_{2 \text { máx }}\left(\mathrm{ml}^{\mathrm{m}} \mathrm{min}^{-1}\right)$ e para $\% \mathrm{VO}_{2 \text { máxLAVv }}$ concluindo que a aptidão física de crianças e adolescentes com excesso de peso é quantitativamente reduzida.
Na literatura estudada, poucos trabalhos mostraram valores das variáveis relacionadas à $F C\left(\mathrm{FC}_{\mathrm{LAV}}, \mathrm{FC}_{\text {máx }}\right.$ e \% FC máxLAV), principalmente ao nível submáximo do teste cardiopulmonar de exercício em esteira. Embora as variáveis citadas acima não tenham apresentado diferenças significativas entre os grupos deste estudo, a resposta fisiológica da $\mathrm{FC}_{\text {máx }}$ está concordante com outros trabalhos ${ }^{6,7,18,19,22,23}$ que compararam adolescentes com excesso de peso e com peso normal, e que encontraram resultados semelhantes na comparação entre os grupos. No presente estudo os adolescentes com excesso de peso não apresentaram diferenças para $\mathrm{FC}_{\text {máx }}$ quando comparados àqueles com peso normal (201 bpm \pm 9 vs 198 bpm \pm 7 ), porém, alguns autores sugerem que a $\mathrm{FC}_{\text {máx }}$ sofre influência da obesidade ${ }^{8,9}$. Nesse sentido, Norman et al. ${ }^{9}$ verificaram que a $\mathrm{FC}_{\text {máx }}$ foi menor nos adolescentes obesos quando comparados aos não obesos, tanto no cicloergômetro (186 bpm \pm 13 vs $196 \mathrm{bpm} \pm 11$; respectivamente), como no teste de caminhada/ corrida de 12 minutos (175 bpm \pm 18 vs 197 bpm \pm 17 ; respectivamente), sugerindo que tanto o descondicionamento quanto a obesidade podem reduzir a $\mathrm{FC}_{\text {máx }}$ em adolescentes com excesso de peso.

Para a $\mathrm{FC}_{\mathrm{LAV}}$ os resultados de Prado et al. ${ }^{24}$ mostraram valores de crianças e adolescentes com peso normal mais altos quando comparados aos adolescentes do GPN deste estudo (159 bpm \pm 5 vs $139 \mathrm{bpm}$ \pm 20 , respectivamente). Essa diferença de valores pode estar relacionada à faixa etária que o presente estudo abrange (10 a 14 anos; média 12,1 $\pm 1,2$ para o GPN), enquanto que em Prado et al..$^{24}$ a média de idade é de 10,2 $( \pm 0,2)$. Como a $F C_{\text {máx }}$ depende da idade cronológica ${ }^{19}$, deduz-se que a $\mathrm{FC}_{\mathrm{LAV}}$ tenha o mesmo comportamento fisiológico.

Neste estudo, utilizamos a velocidade no LAV $(\mathrm{km} / \mathrm{h})$ como variável relacionada à carga de trabalho. Contudo, na literatura são utilizados também outros parâmetros, como: tempo total do teste ${ }^{19}$, distância percorrida em determinado tempo ${ }^{9}$, cálculos para análise de eficiência do trabalho $0^{4,25}$, frequência de passada ${ }^{25}$. Nesse sentido, tornou-se difícil a comparação dos resultados deste estudo com os de outros trabalhos em termos de valores, principalmente porque muitos deles avaliaram apenas os valores máximos do teste de esforço, utilizaram o cicloergômetro 5,9,19,20,26 e/ou trabalharam com diferentes protocolos de teste.

Assim, de maneira geral, ao analisar adolescentes com diferentes graus de IMC de ambos os sexos observou-se que aqueles que são classificados como obesos demonstraram menor tempo de teste na esteira ${ }^{6,7,23}$, menor carga de trabalho no cicloergômetro ${ }^{19}$ (Watts), menor distância percorrida ${ }^{9}$ (metros), quando comparados aos adolescentes com peso normal. Porém, os estudos citados anteriormente avaliaram a carga apenas no pico do teste cardiopulmonar de exercício. No caso deste estudo, a carga de trabalho avaliada no LAV (Vel LAV) foi menor no GEP quando comparado ao GPN, concordante com os dados encontrados na literatura para o máximo do teste de esforço. Norman et al. ${ }^{9}$ sugeriram que esse comportamento é atribuído à diminuição da eficiência mecânica devido ao alto custo metabólico durante o exercício.

\section{CONCLUSÃO}

Nossos dados mostram que os adolescentes com excesso de peso apresentam uma aptidão cardiorrespiratória prejudicada em nível submáximo de desempenho no teste cardiopulmonar de exercício, quando comparados aos adolescentes com peso normal. Nesse sentido, observamos que avaliar e analisar a aptidão cardiorrespiratória no LAV traz informações importantes para a interpretação das diferenças entre grupos de adolescentes de acordo com o IMC. A avaliação das variáveis cardiorrespiratórias obtidas no LAV a partir do teste cardiopulmonar de exercício permite que os adolescentes, principalmente aqueles com excesso de peso, tenham seus resultados analisados sem, necessariamente, atingirem o esforço máximo durante a realização do teste, 
tornando possível a prescrição de exercícios físicos em intensidades mais apropriadas para essa população, o que contribuirá para o aumento da demanda energética, auxiliando nas intervenções de tratamento e prevenção do excesso de peso.

\section{AGRADECIMENTOS}

Os autores agradecem ao Fundo de Apoio à Ciência e Tecnologia
(FACITEC) da Prefeitura Municipal de Vitória, ES e à Fundação de Amparo à Pesquisa do Espírito Santo (FAPES) pelas bolsas outorgadas, e ao Prof. Dr. Anabel Nunes Rodrigues.

Todos os autores declararam não haver qualquer potencial conflito de interesses referente a este artigo.

\section{REFERÊNCIAS}

1. WHO, World Health Organization. Preventing chronic diseases: a vital investment. WHO Global Report. 2005.

2. IBGE. Pesquisa de Orçamentos Familiares 2008-2009: Antropometria e Estado Nutricional de Crianças, Adolescentes e Adultos no Brasil. IBGE 2010.

3. Al-Hazzaa HM. Development of maximal cardiorespiratory function in Saudi boys. Saudi Med J. 2001;22(10):875-81

4. Cooper DM, Weiler-Ravell D, Whipp BJ, Wasserman K. Aerobic parameters of exercise as a function of body size during growth in children. J Appl Physiol. 1984;56:628-34.

5. Ferrari GL, Bracco MM, Matsudo VK, Fisberg M. Cardiorespiratory fitness and nutritional status of schoolchildren: 30-year evolution. J Pediatr, 2013;89:366-73.

6. Prado DM, Silva AG, Trombetta IC, Ribeiro MM, Nicolau CM, Guazzelli IC, et al. Weight Loss Associated with Exercise Training Restores Ventilatory Efficiency in Obese Children. Int J Sports Med. 2009;30:821-6.

7. Zanconato S, Baraldi E, Santuz P, Rigon F, Vido L, Dalt LD, et al. Gas exchange during exercise in obese children. Eur J Pediatr. 1989;1148:614-7.

8. Loftin M, Sothern M, Trosclair L, O'hanlon A, Miller J, Udall J. Scalin $\mathrm{VO}_{2}$ peak in obese and non-obese girl. Obes Res. 2001;9(5):290-6

9. Norman AC, Drinkard B, McDuffie JR, Ghorbani S, Yanoff LB, Yanovski JA. Influence of Excess Adiposity on Exercise Fitness and Performance in Overweight Children and Adolescents. Pediatrics. 2005;115:690-6.

10. Perez AJ, Carletti L. Identificação do limiar anaeróbico ventilatório em crianças e adolescentes: revisão da literatura. Rev Bras Cineantropom Desempenho Hum. 2012;14(3):343-52

11. Washington RL. Why do girls use less oxygen during exercise than boys? Cause or effect of decreased work. Chest. 2000;117(3):619-20

12. Rodrigues NA, Perez AJ, Carletti L, Bissoli NS, Abreu GR. Maximum oxygen uptake in adolescents as measured by cardiopulmonary exercise testing: a classification proposal. J Pediatr. 2006;82:426-30.

13. Rodrigues NA, Perez AJ, Carletti L, Bissoli NS, Abreu GR. The association between cardiorespiratory fitness and cardiovascular risk in adolescents. J Pediatr. 2007:83(5):429-35.

14. Rowland TW, Delaney BC, Siconolfi SF. Athlest's Heart in Prepubertal Children. Pediatrics. 1987;79(5):800-04
15. WHO, Word Health Organization. Growth reference data for 5 - 19 year. 2007. Disponível em <http:// www.who.int/growthref/en/>. Acesso em: 26 set. 2013

16. Rowland TW, Cunningham LN. Oxygen uptake plateau during maximal treadmill exercise in children Chest. 1992;101:485-9.

17. Beaver WL, Wasserman K, Whipp BJ. A new method for detecting anaerobic threshold by gas exchange. J Appl Physiol. 1986;60:2020-7

18. Gomes KB, Carletti L, Perez AJ. Limiar anaeróbico ventilatório em adolescentes: um estudo das variáveis cardiorrespiratórias de adolescentes com peso normal, sobrepeso e obesidade. Saarbrücken: Novas Edições Acadêmicas, 2013.

19. Milano GE, Leite N. Comparação das Variáveis Cardiorrespiratórias de adolescentes obesos e não obesos em Esteira e Bicicleta Ergométrica. Rev Bras Med Esporte. 2009;15(4):251-4.

20. Salvadego D, Lazzer S, Busti C, Galli R, Agosti F, Lafortuna C, et al., Gas exchange kinetics in obese adolescents.Inferences on exercise tolerance and prescription. Am J Physiol Regul Integr Comp Physiol. 2010;299:R1298-R1305.

21. Bar-Or O. Pediatric Sports Medicine for Practitioner: From Physiologic Principles to Clinical Applications. New York: Springer, 1983

22. Carletti L, Rodrigues NA, Perez AJ, Vassallo DV. Resposta da Pressão Arterial ao Esforço em Adolescentes: Influência do Sobrepeso e Obesidade. Arq Bras Cardiol. 2008:91(1):25-30.

23. Goran M, Fields DA, Hunter GR, Herd SL, Weinsier RL. Total body fat does not influence maximal aerobic capacity. Int J Obes. 2000;24:841-8.

24. Prado DM, Braga AMFW, Rondon MUP, Azevedo LF, Matos LDNJ, Negrão CE, et al. Comportamento Cardiorrespiratório em Crianças Saudáveis durante o Exercício Progressivo Máximo. Arq Bras Cardiol. 2010;94(4):493-9.

25. Rowland TW, Cunningham LN, Martel L, Vanderburgh P, Manos T, Charkoudian N. Gender Effects on Submaximal Energy Expenditure in Children. Int J Sports Med. 1997;18:420-5.

26. Giardini A, Odendaal D, Khambadkone S, Derrick G. Physiologic decrease of ventilatory response to exercise in the second decade of life in healthy children. Am Heart J. 2011;161:1214-9. 